\title{
The Threat of Zika Virus in Sub-Saharan Africa - The Need to Remain Vigilant
}

\author{
Vito Baraka ${ }^{1,2}$ and Eliningaya J. Kweka ${ }^{3,4 *}$ \\ ${ }^{1}$ Tanga Research Centre, National Institute for Medical Research, Tanga, Tanzania, ${ }^{2}$ Global Health Institute, Gouverneur \\ Kinsbergen Centrum, University of Antwerp, Wilrijk, Belgium, ${ }^{3}$ Division of Livestock and Human Diseases Vector Control, \\ Tropical Pesticides Research Institute, Arusha, Tanzania, ${ }^{4}$ Department of Medical Parasitology and Entomology, School of \\ Medicine, Catholic University of Health and Allied Sciences, Mwanza, Tanzania
}

Keywords: arboviral infections, Zika virus, diagnosis surveillance and control, sub-Saharan Africa, commentary

\section{BACKGROUND}

News of the recent outbreak of Zika virus (ZIKV) disease in South America, North America, and Europe has generated great interest in the scientific community and general public like $(1,2)$. The disease is caused by the Zika virus (ZIKV), a mosquito-borne Flavivirus transmitted mainly by Aedes aegypti and Aedes albopictus, mosquitoes that also transmit other viral infections, including dengue virus (DENV), chikungunya virus (CHIKV), and yellow fever virus (YFV) (2). Zika virus was iso-

Edited by:

Jorg Heukelbach, Universidade Federal do Ceará,

Brazil

Reviewed by: Aimee Ferraro, Walden University,

Luciano P. G. Cavalcanti, Universidade Federal do Ceará,

Brazil

*Correspondence: Eliningaya J. Kweka kwekae@tpri.or.tz

Specialty section:

This article was submitted to Infectious Diseases, a section of the journal Frontiers in Public Health

Received: 08 April 2016 Accepted: 16 May 2016

Published: 31 May 2016

Citation:

Baraka V and Kweka EJ (2016) The Threat of Zika Virus in Sub-Saharan Africa - The Need to Remain Vigilant. Front. Public Health 4:110. doi: 10.3389/fpubh.2016.00110 lated for the first time in Rhesus monkey in 1947 in Zika forest in Uganda and since then, evidence of seroprevalence of ZIKV infection in human has been documented in several African countries (3-5). However, to date, the virus has not been considered a serious threat in the region. Symptoms of Zika virus disease are very similar to those of dengue and chikungunya and include fever, rash, joint pain, or conjunctivitis (6). Furthermore, most of the infections remain asymptomatic; thus, majority of the cases are either misdiagnosed or not detected at all. Worryingly, the recent pandemic in South America has associated ZIKV infection with microcephaly, a condition that results in small heads and underdeveloped brains in infants and neurological complication (Guillain-Barré syndrome); yet, no specific treatment or vaccine for the disease exists $(1,7)$. The incidences of ZIKV infections are escalating at alarming rates in South, North America, and in Europe and potentially threatening countries in sub-Saharan Africa if migration might play a role in both directions. Increasing urbanization, poor urban planning, changes in climatic factors, and the availability of favorable microecological condition suitable for Aedes mosquitoes breeding in sub-Saharan Africa are among factors that escalate mosquito abundance. In the face of such potential threat, there is a need for vigilance and establishment of preparedness measures before a Zika pandemic hits the continent. Such a pandemic would pose overwhelming cost burdens to the health systems and potentially compromise the achievement of the sustainable development goals (SDGs). In this letter, we wish to highlight measures that we believe would be effective in setting up countries' preparedness response and surveillance systems to address the potential Zika virus disease threat in sub-Saharan context.

First, there is need for capacity strengthening with focus on the laboratory facilities and human resources to be able to implement epidemiological surveillance and disease control carry out accurate diagnosis and offer quality case management during outbreaks. The establishment of guidelines for all these aspects of disease management would need to be developed. There is need to support the setting up public health laboratories and strengthening of the existing ones to be able to conduct epidemiological surveillance and sophisticated molecular diagnosis that relies on polymerase chain reaction (PCR) or real-time PCR (RT-PCR) based assays. Currently, there is no ZIKV rapid diagnostic test available at the point of care. Therefore, it is important that health-care professionals 
are trained on case diagnosis and management approaches. This has to be in parallel with the improved capacity of laboratories to exclude other severe conditions, such as malaria and bacterial infection. Regional and cross-border networks, such as the East Africa Public Health Laboratory Networking (EAPHLNP), should be strengthened to fill the gaps and the model emulated in other African countries. In this era of increased mobility between countries, the need for regional coordination in sharing of virologic/serotype and vector surveillance data should be underscored. Additionally, there is a shortage of entomologists at regional and district levels to provide technical support in mosquito vector identification and dynamics, which is critical for vector surveillance and control. This shortage of qualified entomologists with technical field expertise is well documented (8). We therefore suggest that serious consideration be given to the training of entomologists so as to fill this gap.

Second, there is a need to raise community awareness and to educate the public on measures that they can put in place to avoid mosquito bites and reduce mosquito breeding habitats. A. aegypti are container breeders and integrated approaches that require close community engagement are necessary for their effective control. Community awareness and education will contribute toward the adjustment of risky behavior, such as the failure to cover water storage containers that are in use and improper disposal of old water containers and used car tires. The media should be granted the opportunity by governments to take the lead in supporting community awareness and education efforts on all matters related to epidemiology and control of Zika virus disease more than is currently happening (2).

Third, the role of global travel in the emergence and re-emergence of disease diseases cannot go unnoticed. There is already risk for transmission of Zika virus disease to sub-Saharan African countries in Cape Verde and other regions $(3,9)$. Expanding global travel and the shipping industry contribute significantly in the transportation of asymptomatic individuals (10). Strong incentives are needed for surveillance to prevent re-infestation of the Zika virus in sub-Saharan Africa. Attention is particularly needed in the main entry points such as the airports and seaports that are the main gateway from the infected areas. There is need for ministries of health and respective authorities to issue travel alerts and guidance to those visiting to ZIKV-risk countries. For example, pregnant women in any trimester should be advised to highly consider postponing travel and individuals who must visit such countries provided with guidelines and recommendations on the symptoms to look out for and immediately report to the health-care professional on the onset of such symptoms. Also, guidelines and recommendations on personal protection measures to avoid human-mosquito contact, such as the use of repellants and wearing long sleeve clothes, should be emphasized.

Fourth, several key research issues need to be addressed. It will be important to evaluate the role of potential non-human primates in maintaining transmission and/or serving as ZIKV reservoirs. Other proposed routes of transmission, including sexual and maternal (Figure 1), also need further investigation as this will have implications for the epidemiology of the disease. In addition, given that there are no preventative or therapeutic vaccines and point of care diagnostic tests for Zika virus disease, it is important that financial resources to accelerate the discovery and clinical testing of these tools be urgently set aside and made available. With regard to vector control, strategies relevant to the local context are urgently needed to support effective preventive and control measures. The increasing role of climatic factors in relation to Aedes mosquito dynamics also needs further exploration as the changes in global temperatures and weather patterns could impact the transmission and spread of the virus. Possible consequences of coinfections between dengue serotypes

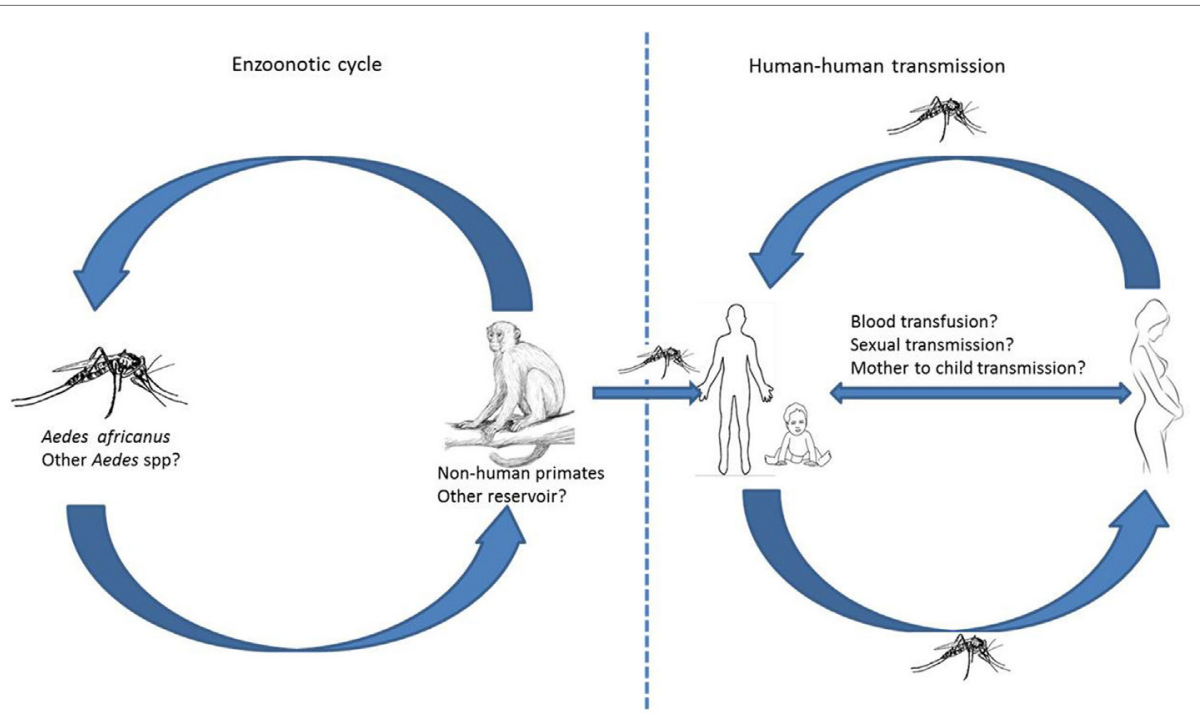

FIGURE 1 | Zika virus is transmitted mainly by the Aedes aegypti mosquitoes, which is widespread in urban and peri-urban areas. The zoonotic is known to occur between human and non-human primates. The role of other reservoir and sexual transmission is still unconfirmed. 
(DENV 1-4) and ZIKV virus or coinfection between ZIKV and other prevalent infections in the continent, such as malaria and HIV, also need to be understood. Furthermore, the implication of different ZIKV serotypes in vulnerable groups' particularly pregnant women and children are yet to be understood. With Africa increasingly opening up to the rest of the world due to human migration associated with tourism and business, it is imperative for countries to remain vigilant regarding the threat of the expanding arboviral infections.

The re-emergence and spread of arboviral infections could lead to devastating consequences on the human population, the health-care system and economic progress in the continent. It is

\section{REFERENCES}

1. Fauci AS, Morens DM. Zika virus in the Americas - yet another arbovirus threat. N Engl J Med (2016) 374(7):601-4. doi:10.1056/NEJMp1600297

2. Gyawali N, Bradbury RS, Taylor-Robinson AW. The global spread of Zika virus: is public and media concern justified in regions currently unaffected? Infect Dis Poverty (2016) 5(1):1-6. doi:10.1186/s40249-016-0132-y

3. Grard G, Caron M, Mombo IM, Nkoghe D, Mboui Ondo SM, Jiolle D, et al. Zika virus in Gabon (Central Africa) - 2007: a new threat from Aedes albopictus? PLoS Negl Trop Dis (2014) 8(2):e2681. doi:10.1371/journal.pntd.0002681

4. Berthet N, Nakouné E, Kamgang B, Selekon B, Descorps-Declère S, Gessain A, et al. Molecular characterization of three Zika flaviviruses obtained from sylvatic mosquitoes in the Central African Republic. Vector Borne Zoonotic Dis (2014) 14(12):862-5. doi:10.1089/vbz.2014.1607

5. Wang H, Wang S. Zika virus: old rival, new threat. Infect Dis Transl Med (2016) 2(1):10-19.

6. Dick G, Kitchen S, Haddow A. Zika virus (I). Isolations and serological specificity. Trans $R$ Soc Trop Med Hyg (1952) 46(5):509-20. doi:10.1016/0035-9203(52)90042-4

7. Oehler E, Watrin L, Larre P, Leparc-Goffart I, Lastere S, Valour F, et al. Zika virus infection complicated by Guillain-Barré syndrome - case report, crucial that countries establish harmonized and robust vector control and surveillance systems, which will include the setting up of regional preparedness plans in response to mosquito-borne viruses and investing in capacity building as well as creating community awareness. Investing in research in the development and validation of tools and strategies for the control of the viruses and in understanding of their epidemiology will also be critical.

\section{AUTHOR CONTRIBUTIONS}

Both authors, VB and EK contributed equally to the drafting and approved the final manuscript for submission.

French Polynesia. Euro Surveill (2014) 19(9):20720. doi:10.2807/1560-7917. ES2014.19.9.20720

8. Mnzava AP, Macdonald MB, Knox TB, Temu EA, Shiff CJ. Malaria vector control at a crossroads: public health entomology and the drive to elimination. Trans R Soc Trop Med Hyg (2014) 108(9):550-4. doi:10.1093/trstmh/tru101

9. Attar N. Zika virus circulates in new regions. Nat Rev Micro (2016) 14(2):62. doi:10.1038/nrmicro.2015.28

10. Nah K, Mizumoto K, Miyamatsu Y, Yasuda Y, Kinoshita R, Nishiura H. Estimating risks of importation and local transmission of Zika virus infection. PeerJ (2016) 4:e1904. doi:10.7717/peerj.1904

Conflict of Interest Statement: The authors declare that the research was conducted in the absence of any commercial or financial relationships that could be construed as a potential conflict of interest.

Copyright (c) 2016 Baraka and Kweka. This is an open-access article distributed under the terms of the Creative Commons Attribution License (CC BY). The use, distribution or reproduction in other forums is permitted, provided the original author(s) or licensor are credited and that the original publication in this journal is cited, in accordance with accepted academic practice. No use, distribution or reproduction is permitted which does not comply with these terms. 